\title{
El agregado digital en las juventudes indígenas: entre desigualdades y
} representaciones locales

\author{
Oscar Ramos-Mancilla ' \\ http:/ / orcid.org/ 0000-0003-4258-1993 \\ I Universidad Autónoma de Puebla, México. \\ Professor do Instituto de Ciências Sociais e Humanidades.
}

http:/ / dx.doi.org/ 10.1590/ 1981-5344/ 3870

Las infraestructuras de conectividad a internet se han extendido hasta en los poblados indígenas, sin embargo, son básicamente los jóvenes quienes utilizan las tecnologías digitales. Los principales servicios que utilizan son las redes sociales para comunicarse, relacionarse y subir contenidos propios. A partir de un acercamiento etnográfico a tres poblados en México, expongo algunas características de las condiciones de conectividad y de los contenidos que producen y comparten los jóvenes. Dichos contenidos reflejan las interacciones que experimentan entre sus mundos locales y las posibilidades fuera de sus lugares de origen.

Palavras clave: juventude. pueblos indígenas. redes sociales. etnografía.

\section{O agregado digital em jovens indígenas: entre desigualdades e representações locais}


As infraestruturas de conectividade da Internet se estenderam mesmo em aldeias indígenas, no entanto, os jovens são basicamente aqueles que usam tecnologias digitais. Os principais serviços que usam são redes sociais para se comunicar, interagir e fazer upload de seu próprio conteúdo. De uma abordagem etnográfica a três aldeias no México, exponho algumas características das condições de conectividade e os conteúdos que os jovens produzem e compartilham. Esses conteúdos refletem as interações que eles experimentam entre seus mundos locais e as possibilidades fora de seus locais de origem.

Palavras-chave: Juventude. Povos indígenas. Redes sociais. Etnografia.

\section{The digital attached in the indigenous youths: between inequalities and local representations}

Internet connectivity infrastructures have extended even in indigenous villages; however, young people are basically those who use digital technologies. The main services they use are social media to communicate, interact and upload their own content. From an ethnographic approach to three villages in Mexico, I expose some characteristics of the conditions of connectivity and the contents that young people produce and share. These contents reflect the interactions they experience between their local worlds and the possibilities outside of their places of origin.

Keywords: Youth. Indigenous peoples. Social media. Ethnography.

Recebido em 25.02.2019 Aceito em 31.03.2020

\section{I ntroducción}

Los estudios de internet se han enfocado en los impactos que generan las nuevas dinámicas en la comunicación, en la realización de tareas y trabajos, en el mantenimiento de comunidades transnacionales, en los usos sociales, entre otros (DUTTON, 1999; FEENBERG; FRIESEN, 
2012). Pero, las exploraciones se han concentrado en contextos más tecnificados, donde hay mayor acceso a las tecnologías digitales, con mayor ancho de banda y conectividad constante. Sin embargo, hay otros lugares que también están experimentando la conectividad a internet y el uso de diferentes tecnologías digitales como es el caso de los pueblos indígenas (ACOSTA, 2017; DYSON et al., 2007; SRINIVASAN, 2006).

Por otro lado, los jóvenes han captado una atención especial porque se les suele asignar una facilidad para integrar las tecnologías digitales en sus actividades cotidianas y de entretenimiento (BENNET; ROBARDS, 2014; BOYD, 2014). Precisamente, en los poblados indígenas son las y los jóvenes quienes tienen mayor acceso a las tecnologías digitales, en parte porque se han vinculado hacia la educación y con ello hacia otros servicios y ámbitos que, en general, han conformado espacios de socialización de jóvenes lo que reproduce y fomenta el uso de tecnologías digitales. Este ámbito, lo digital, se suma e incide en la multiplicidad de las situaciones actuales de los pueblos indígenas, por lo que se hace relevante indagar las repuestas por parte de los jóvenes ante sus nuevas situaciones de vida, y precisamente las redes sociales digitales son un recurso que hace posible y amplía la observación.

Así, considero que lo indígena se va resignificando constantemente, en medio de interacciones e intercambios diversos. La presencia de las y los jóvenes como sujetos sociales, con sus propias lógicas y entendimientos, propician reelaboraciones en lo indígena y en las ideas de comunidad (RAMOS, 2015a). En este sentido, el objetivo de este texto es exponer que dichas reelaboraciones pasan por, en este caso relacionado al espacio digital, las desigualdades en el acceso a las tecnologías de información y a las representaciones de lo local que se expresan en redes sociales. Con ello, la juventud indígena con sus interacciones diferenciadas, propicia que la vida en la comunidad contenga referentes mucho más heterogéneos y en distintas escalas.

\section{Algunas ideas en torno a la juventud indígena}

La juventud, como categoría para observar expresiones y socialización de determinadas experiencias de vida (FEIXA, 1998; URTEAGA, 2011; WINOCUR, 2006), tiene presencia en los pueblos indígenas tanto en sus territorios como fuera de estos (CLIFFORD, 2007; KUMMELS, 2017), y con múltiples relaciones y prácticas que han comenzado a reflejarse en los acercamientos de investigaciones sociales (PÉREZ RUÍZ; VALLADARES, 2014; MESEGUER, 2012; URTEAGA; GARCÍA, 2015). La marcada presencia de este sector de la población ${ }^{1} e n$ los

\footnotetext{
${ }^{1}$ En De jóvenes, bandas y tribus (1998), Carles Feixa hacía notar lo poco estudiado de la juventud indígena, particularmente en México dondeel indigenismo había generado una tradición institucional y académica hacia
} 
poblados indígenas y rurales responde, juntoal propio crecimiento demográfico, a circunstancias externas que han incidido en lo local y viceversa, como es el incremento de espacios educativos solicitados por las mismas poblaciones y con ello espacios de socialización juveniles, además de los medios de comunicación que proyectan estilos de vida y modelos de conducta (FEIXA, 1998, p. 138-139; PÉREZ RUÍZ; VALLADARES, 2014).

En este sentido, en la compilación que hacen Pérez Ruiz y Valladares (2014) se sugiere que los jóvenes interactúan en múltiples espacios sociales y culturales, en donde intervienen las experiencias de género, las familias, los recursos económicos y el acceso a servicios, los consumos de medios de comunicación, entre otros. Este es el punto que me parece más evocativo con relación a las experiencias que observé durante el trabajo de campo, puesalrededor de la consolidación de la presencia de la juventudesgeneranideas y representacionesde lo que es la juventud en las poblaciones y comunidades indígenas, las cuales se intercalan, contraponen, complementan, con las representaciones propias y variadas de las y los jóvenes de sí mismos y de los entornos en los que viven. Es en estas representaciones heterogéneas de sus experiencias como jóvenes en pueblos indígenas donde, precisamente, el agregado de lo digital es relevante. Se trata de un ámbito de las relaciones sociales a las que acceden los jóvenes en este momento histórico y que les diferencia de las generaciones que les preceden, de la misma manera, es un espacio público en el que se comparten algunos fragmentos de sus identidades, intereses y preocupaciones, por lo que al mismo tiempo que se manifiestan también se reflejan o contrastan.

Tal como lo han reportado algunos trabajos revisionistas de la juventud indígena (CORTÉS; HERNÁNDEZ, 2016; PÉREZ RUÍZ, 2011; URTEAGA; GARCÍA, 2015; ZAPATA; HOYOS, 2005), hay algunos retos al tratar de definir dicha categoría.Primero, los trabajos pioneros de la juventud se centraron en las sociedades urbanas por lo que se deben ajustar a los contextos indígenas; segundo, la identidad cultural vinculada a la etnia y lo indígena suele relacionarse con lo tradicional (aunque cada vez se reconocen las contradicciones y reacomodos), o incluso se piensa de manera homogénea como si las diferencias socioculturales entre los pueblos indígenas se diluyeran; y tercero,los sujetos que se intentan definir son diversos y complejos. Coincidiendo con este panorama y contrastando lo que encontré en el trabajo de campo, considero que la juventud como categoría es dinámica, entre negociaciones de elementos locales que se van transmitiendo (MEAD, 1970) junto con aquellos 
externos que atraviesan a las subjetividades de este sector etario (PÉREZ RUÍZ; VALLADARES, 2014; URTEAGA; GARCÍA, 2015). Así, retomo la estrategia que utilizan Pérez Ruíz y Valladares (2014) de reportar las características de las condiciones y expresiones de los jóvenes en contexto determinados antes que intentar una definición de juventud indígena, pero, desde las cuales se puede ir ampliando y complementando esta categoría.

\section{De lo digital en los pueblos indígenas}

Una tendencia en las investigaciones que abordan las tecnologías de información y comunicación en los pueblos indígenas proviene de haberse enfocado primero en los usos de los medios de comunicación (BUDKA, 2009; GINSBURG, 2008; KUMMELS, 2017). Desde mediados de la década de 1970 la población indígena tuvo acceso a recursos de comunicación y el proceso de apropiación se consideró como una posibilidad para "rebatir a las estructuras de poder que han borrado o distorsionado sus intereses y realidades"2 (GINSBURG et al., 2002, p. 7). Al retomar estos antecedentes considero que, así como los medios de comunicación fueron y son recursos que pueden ser apropiados para construir representaciones y narrativas propias, actualmente sucede con internet porque ahí convergen las versiones digitales de los medios de comunicación.

Por otro lado, a la juventud se le ha adjudicado un acercamiento y facilidad para utilizar recientes tecnologías (BENNETT; ROBARDS, 2015) y, en el momento actual con las tecnologías digitales también se les ha asignado el mismo papel bajo denominaciones como, por ejemplo, la de nativos digitales ${ }^{3}$ (PRESKY, 2001). En el caso de los poblados indígenas, los procesos sociales e históricos que han configurado las diferencias desde las cuales la juventud indígena utiliza las tecnologías digitales, genera que el agregado digital sea un recurso casi exclusivo de estos sectores de la población porque tienen mayores índices de alfabetización que las generaciones anteriores y porque en los recientes años ha aumentado la infraestructura de tecnologías de información y comunicación.

Las interacciones de los jóvenes con los recursos digitales se realizan, principalmente, desde los entornos de las redes sociales o Web 2.0. En estos entornos convergen herramientas que posibilitan otras formas de producir y compartir contenido propio, modificar o interactuar con contenidos que ya están disponibles, o sea, respaldan otras relaciones sociales y producciones culturales que expanden los referentes locales y

\footnotetext{
${ }^{2}$ Traducción propia.

${ }^{3}$ Para una crítica al concepto de nativos digitales se puede leer Bennett et al. (2008).
} 
personales (DOMÍ NGUEZ FIGAREDO, 2012, p. 202). Aunque cabe indicar que estas posibilidades requieren habilidades particulares, desde escribir hasta aquellas que se consideran específicas para utilizar internet (VAN DEURSEN; VAN DIJ K, 2010).

En It's Complicated (2014), danah boyd ${ }^{4}$, indica que las redes sociales digitales son espacios de socialización que los jóvenes se han apropiado con facilidad porque estos servicios les permiten crear contenidos, interactuar, comunicarse y generar comunidades online. Por otro lado, Ramesh Srivinasan (2017) señala que todas estas redes sociales digitales funcionan por la circulación de contenidos, los cuales propician la interacción, pero, la particularidad es que los contenidos no son ofrecidos por los servicios de redes sociales, sino que son las personas usuarias quienes proporcionan los contenidos, o bien quienes las enlazan y comparten desde otros agentes y medios de comunicación.

En este sentido, a través de las redes sociales $y$, específicamente, de los contenidos que generan las y los jóvenes provenientes de poblados indígenas se pueden explorar las representaciones que realizan de sus entornos (MADER, 2007), de sus poblados y de sí mismos, y con ello las ideas en torno a sus comunidades, la juventud, y lo étnico. Así, se suman a lo que Eliete da Silva Pereira (2012) ha expuesto, que la apropiación de tecnologías digitales y las interacciones que propician generan a su vez reelaboraciones étnicas indígenas, las cuales son producidas desde las propias personas en contraste con las imágenes que desde fuera se les ha asignado a los pueblos indígenas (GINSBURG et al., 2002).

\section{Metodología}

La información que se expone en este artículo corresponde a un trabajo cualitativo más amplio que fue resultado de mi investigación doctoral. En este sentido, recojo parte de dicha información (orientada hacia los usos e impactos de internet en poblados indígenas) para ampliar en torno a las desigualdades de acceso a TIC y para presentar diferentes contenidos producidos por las y los jóvenes indígenas en los entornos digitales. El trabajo de campo fue realizado bajo una perspectiva etnográfica y lo dividí en dos momentos. El primer momento lo realicé en tres pobladosde la Sierra Norte de Puebla que cumplieron con dos características, esto es, tenían servicios de conexión a internet como cibercafés o telecentros, y había personas utilizando esos servicios, además de que estuvieran habitados en su mayoría por población indígena. El segundo momento correspondió a las interacciones por medio de redes sociales digitales.

${ }^{4}$ Esta investigadora prefiere que su nombre se escriba en minúsculas. 
En la Sierra Norte de Puebla habita la población mestiza y cuatro grupos étnicos, nahuas, otomíes, tepehuas y totonacos. La mayoría de los servicios públicos aún es un tema pendiente en muchos de los poblados, básicamente en los de menor número de habitantes. En la región hay suficientes vías de comunicación y transporte público, lo que se manifiesta en un flujo constante de personas, de intercambios comerciales y de relaciones regionales. Los tres poblados donde situé el trabajo correspondieron a dos nahuas y uno totonaco, con diferentes características entre sí y con diferentes interrelaciones con los demás poblados de la región. Al realizar las observaciones iniciales me fui percatando que el sector de la población que más utiliza las tecnologías digitales corresponde a las y los jóvenes, por lo que en el primer momento del trabajo de campo las interacciones se fueron concentrando con las personas de entre 15 y 18 años, aunque también me relacioné y realicé entrevistas a quienes estaban a cargo de los puntos de acceso, como los cibercafés o telecentros públicos y comunitarios. Pero, los poblados están compuestos por diferentes personas, así que realicé entrevistas y observación con otros pobladores para ampliar mis percepciones de la vida social local.

El segundo momento del trabajo de campo fue a distancia por medio de los servicios de redes sociales que utilizaban las y los jóvenes (cabe indicar que en el primer periodo identifiqué los servicios que utilizaban). En este sentido, algunas investigaciones (HINE, 2004; MILLER; SLATER, 2000; POSTILL, 2011) ya han señalado la necesidad y conveniencia de complementar los datos que se obtienen en ambos tipos de interacciones. Jenna Burrell indica que el sitio desde donde se realiza la observación puede considerarse como una red heterogénea de relaciones (BURRELL, 2009, p. 182), es decir, las experiencias pueden estar situadas localmente, pero, al mismo tiempo son un cúmulo de diversas conexiones, en este caso algunas online y otras offline.

El traslado hacia el trabajo de campo online tuvo al menos dos particularidades, una fue que la interacción se realizó entre personas que ya nos conocíamos de manera previa a la inmersión digital; la segunda, que internet es lo que las personas hacen de sus posibilidades de exploración y navegación, en este sentido, traté de ubicar los servicios que me permitieran relacionarme con las y los usuarios, en este caso, aunque algunas personas utilizan servicios de microblogueo o canales de videos, entre otros, la mayoría con quienes interactué en la Sierra Norte de Puebla utilizaban la red social Facebook. Las características de esta red social nos permitieron mantener comunicación privada (con el servicio de mensajes privados), colectiva y pública (directamente en los "muros"), así como seguir los enlaces hacia noticias, videos, observar los contenidos que generaban y compartían, y las interacciones que iban surgiendo. A 
partir de esta observación participante fui obteniendo la información que describo más adelante.

La separación e integración de dos momentos del trabajo de campo para el registro de la información fue muy útil para ampliar y cruzar los datos de las personas que participaron en la investigación. Fue, además, un reflejo de los espacios sociales interrelacionados en los que se desenvuelven las personas desde los poblados indígenas, y de manera específica la población joven.

\section{Desigualdades y diferencias constantes}

Las interacciones digitales desde los poblados indígenas requieren de infraestructura, de materialidad para las conexiones, es decir, de antenas repetidoras del espectro, de receptores de las señales, de cables y módems, y entre la población se requiere de aparatos y dispositivos con los cuales conectarse. El panorama actual de acceso a internet en la Sierra Norte de Puebla deviene de la implementación de diferentes proyectos gubernamentales y civiles que desde hace dos décadas intentan reducir la brecha digital, así, en muchos de los poblados indígenas comenzaron a tener conectividad desde telecentros públicos y telecentros comunitarios.Conforme la demanda del servicio fue aumentando y la infraestructura se fue extendiendo, también comenzaron a abrirse cibercafés, y ahora son éstos los principales sitios desde los cuales conectarse. Además, en los recientes años ha incrementado el uso de teléfonos móviles por parte de las y los jóvenes, y aunque este servicio estaba disponible en pocos puntos desde hace más de una década es ahora que muchas de las conexiones digitales se realizan desde estos dispositivos portátiles.

Las posibilidades de conectividad van emparentadas con las personas que son mediadoras del acceso a las tecnologías de información y comunicación. A partir del trabajo de campo pude identificar que las personas que se encargan de los puntos de acceso llegan a influir en la apropiación de las tecnologías digitales, sin distinción de que se trate de un cibercafé o un telecentro ya sea comunitario o público. Jeanna Burrell (2011) identificó que antes de comenzar a utilizar una tecnología suele haber ideas previas de las posibilidades de uso, y que suelen ser las personas que consideramos expertas quienes tienen mayor influencia en los primeros acercamientos a las TICs. Así, en campo fui observando que ciertamente las personas encargadas de los puntos de acceso eranlas primeras consultadas para la resolución de problemas vinculados con las tecnologías digitales. Pero, en los casos donde estas personas estaban comprometidas o involucradas con las problemáticas locales, la mediación con las tecnologías era distinta, se buscaban más los usos sociales y que el acceso fuera más generalizado. 
Así, las relaciones en los espacios digitales desde los poblados indígenas deben considerarse a partir de que el acceso a las tecnologías de información y comunicación se realiza aún de manera desigual y al mismo tiempo es heterogénea. Es decir, en cada poblado se pueden encontrar contextos diversos por las condiciones materiales que permiten la conectividad y por las mediaciones que realizan las personas encargadas de los puntos de acceso y conexión.

\section{Representaciones locales en las redes sociales}

Los servicios de redes sociales facilitan la interacción entre las personas y que se puedan agregar contenidos, pero estos servicios no generan ninguna dinámica por sí mismos, es necesario que las personas publiquen, escriban, hagan clic con el cursor, suban una fotografía, compartan un enlace, utilicen un filtro, lean, vean, escuchen, que realicen algo. Esta serie de actividades esta contextualizada por los entornos y subjetividades de las personas que las realizan, en este caso se trata de las y los jóvenes indígenas, y desde las cuales se puede caracterizar un momento específico de la juventud indígena. El contenido que producen es amplio, sin embargo, expongo algunos ejemplos que permiten observar que los jóvenes indígenas están interesados en las actividades y asuntos de sus lugares de origen y de sus comunidades, quizá no como esperan las generaciones adultas, sino más bien desde las juventudes que son.

\section{Pasado y memoria}

Uno de los temas que es común encontrar en las publicaciones de los perfiles de redes sociales es el pasado, la recuperación de la memoria y el arraigo a un lugar de origen. Dos ejemplos pueden ilustrar este interés por parte de la población joven y sus maneras de expresarlo con apoyo de las tecnologías digitales. En el poblado totonaco de Ixtepec se realizó una reunión convocada por un perfil de Facebook con el mismo nombre del pueblo (éste funcionaba como un foro público para tratar asuntos locales), el objetivo fue conocernos entre algunos usuarios que habíamos estado escribiendo nuestras opiniones en el muro de aquel perfil. En esa reunión una de las jóvenes comentó que deberíamos aprovechar los recursos tecnológicos, como grabadoras y cámaras digitales, para ir a realizar entrevistas a las personas ancianas porque eran quienes podían relatar sucesos ocurridos en décadas anteriores y porque conocían cuentos e historias que les habían relatado sus abuelos, agregó que le preocupaba que se desaprovechara esa memoria porque las siguientes generaciones se perderían partes de la historia del pueblo. Un aspecto relevante de su intervención es que ella estaba expresando su opinión sobre el valor de las historias de su lugar de origen frente a un grupo, por su parte los asistentes reaccionaron y agregaron que podían 
hacer una página web para alojar todo ese material y que serviría como un gran depósito de relatos del pueblo y sus habitantes.

El segundo ejemplo proviene de una práctica digital realizada por un joven del pueblo nahua de Huahuaxtla. Polo, quien recién había terminado el bachillerato y estaba trabajando en la presidencia auxiliar del poblado, encontró en el archivo de la presidencia una serie de fotografías antiguas y las escaneó bajo el impulso de conservar las imágenes (no las fotografías en términos materiales). Después, publicó un álbum de fotos al que tituló "baúl de los recuerdos" en la red social Facebook, y al preguntarle por su intensión al compartirlas comentó: "aunque quizá no sean las [fotografías] originales, pero sin duda reflejan el pasado de una población sus costumbres y tradiciones." (Polo. Entrevista virtual, marzo de 2015). 
Imagen 1. Referentes del pasado, serie de fotografías antiguas.

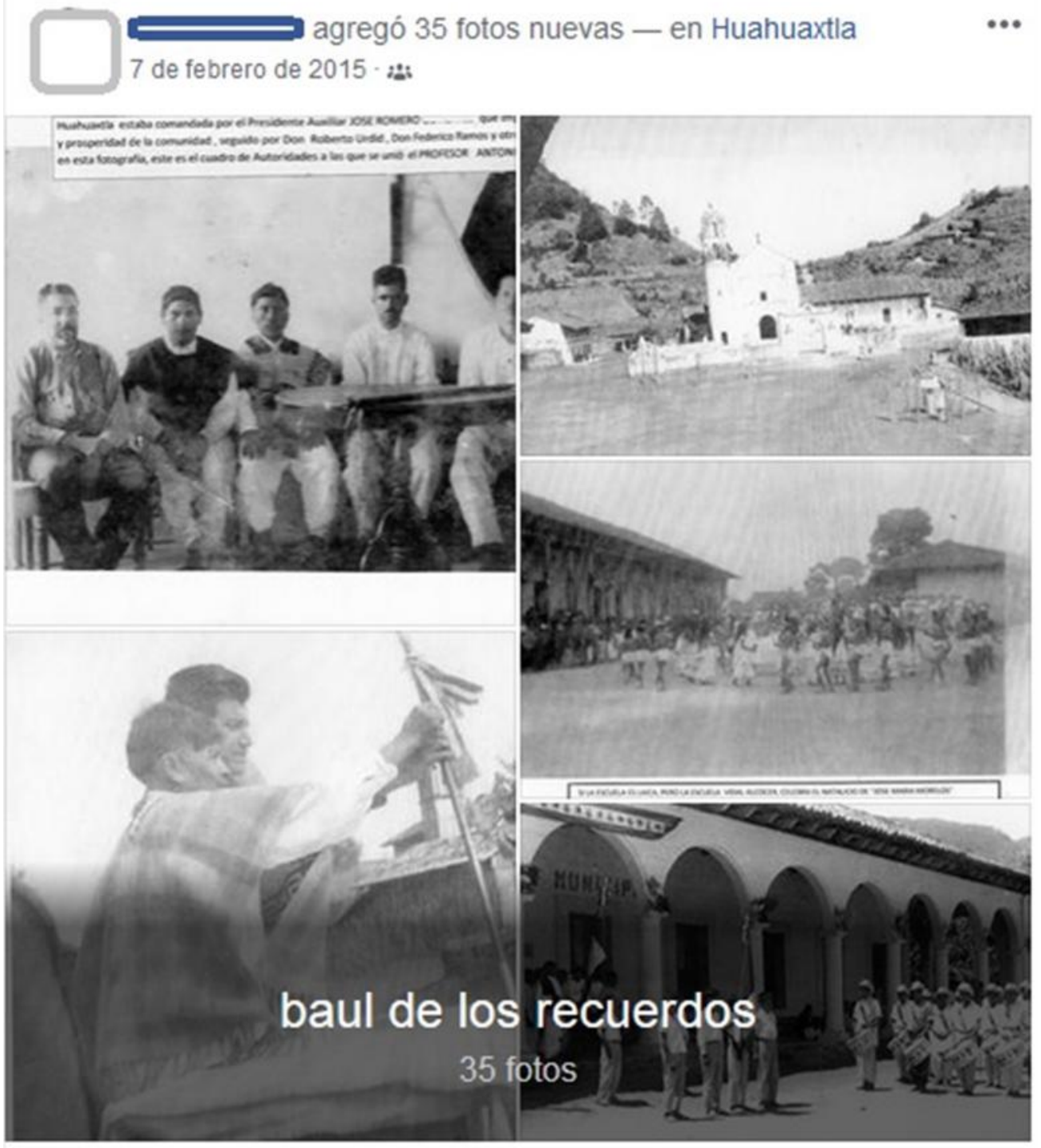

(1) Tú, 1 _ y 9 personas más 13 comentarios

Fuente: Usuario de Facebook, Polo, cortesía.

\section{Fiestas y celebraciones}

Otro de los principales temas que se representan en las redes sociales son las fiestas y celebraciones. Las ferias en conmemoración a los santos de cada pueblo son momentos que se viven de manera distinta según las generaciones, para las personas adultas la feria del pueblo es una responsabilidad, un deber ser hacia la comunidad, mientras que para los jóvenes son momentos de fiesta y diversión. Aun así, para éstos últimos adquiere relevancia participar en las actividades que forman parte de la vida en la comunidad (MADER, 2007), lo cual puede observarse en los contenidos que se comparten por las redes sociales, como fotografías y videos de algunos momentos, además de dar "me gusta" a los enlaces donde se habla de la feria. 
Imagen 2. Panorámica hacia el mercado y la feria.

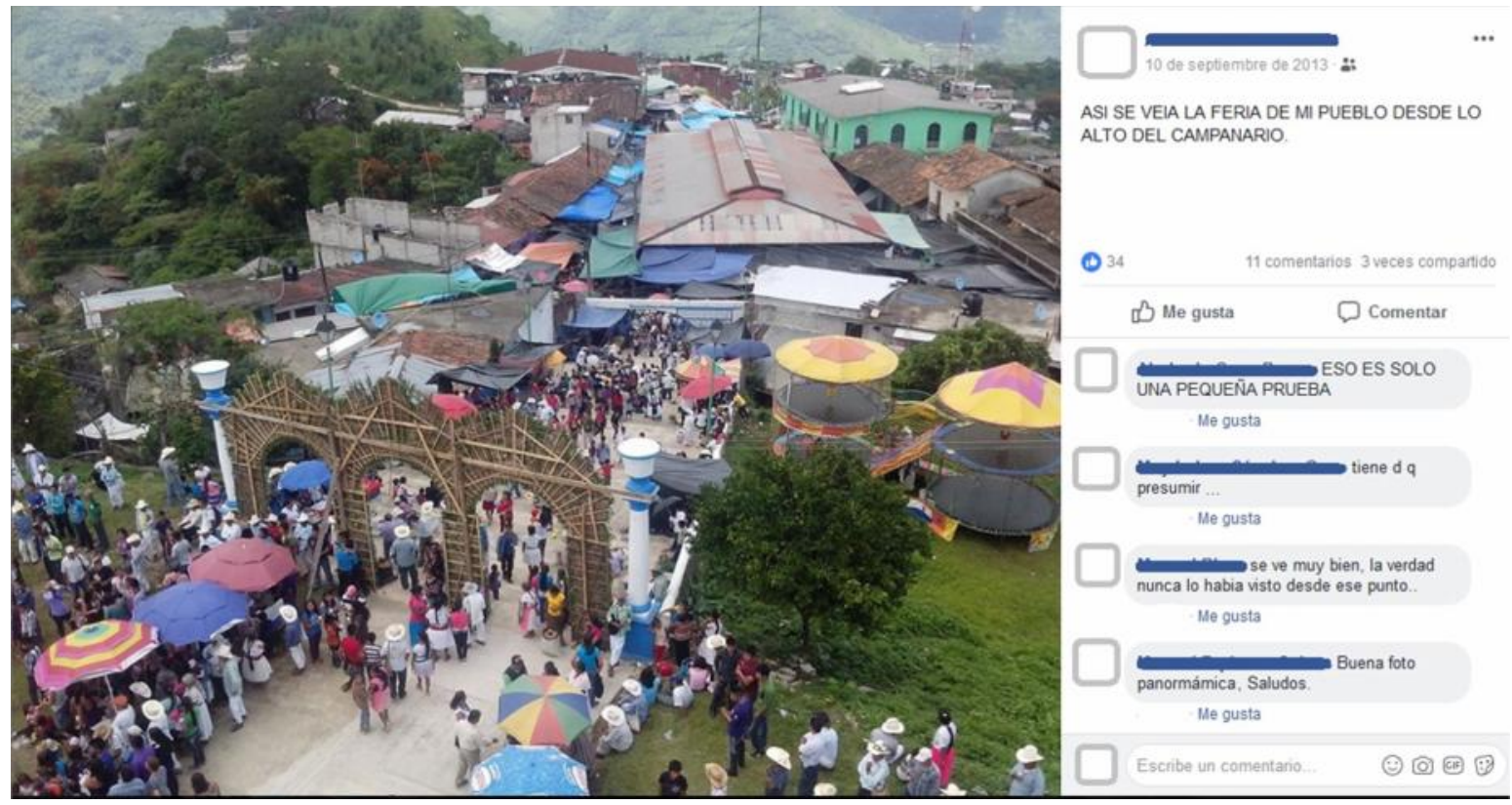

Fuente: Captura de imagen propia a partir del perfil de redes sociales de Luis, cortesía.

Además de la fiesta patronal, durante el año hay otras fechas que también generan espacios de socialización para los jóvenes. Entre las fechas que siguen siendo importantes en los poblados donde trabajé estaban aquellas que corresponden a ciclos rituales como el 3 de mayo, el 24 de junio, año nuevo, entre otras. Mientras que otras fechas son más ambiguas porque combinan diferentes elementos de manera sincrética, pero, quizá son más importantes para los jóvenes porque permiten mayores espacios de juego y socialización, por ejemplo, el Día de Muertos, el Carnaval, y las Posadas.

\section{Entorno local y territorio}

Otro conjunto de actividades que los jóvenes indígenas realizan son los paseos por la Sierra, a veces son lugares cercanos y en otras son más alejados, en ocasiones son a ríos, grutas, miradores, cerros, u otros poblados. En conjunto, esta práctica les va dando un conocimiento espacial a partir de los lugares en donde viven, reconocen lugares, establecen una relación con el territorio, además, en algunas ocasiones también es un momento de socialización con familiares o con amistades. La posibilidad de tener un teléfono móvil o una cámara digital les permite realizar registros de esos recorridos y posteriormente subirlos a las redes sociales, y establecer otras relaciones con sus contactos quienes les comentan sus fotografías o videos. 
Imagen 3. Los pueblos desde diferentes perspectivas.

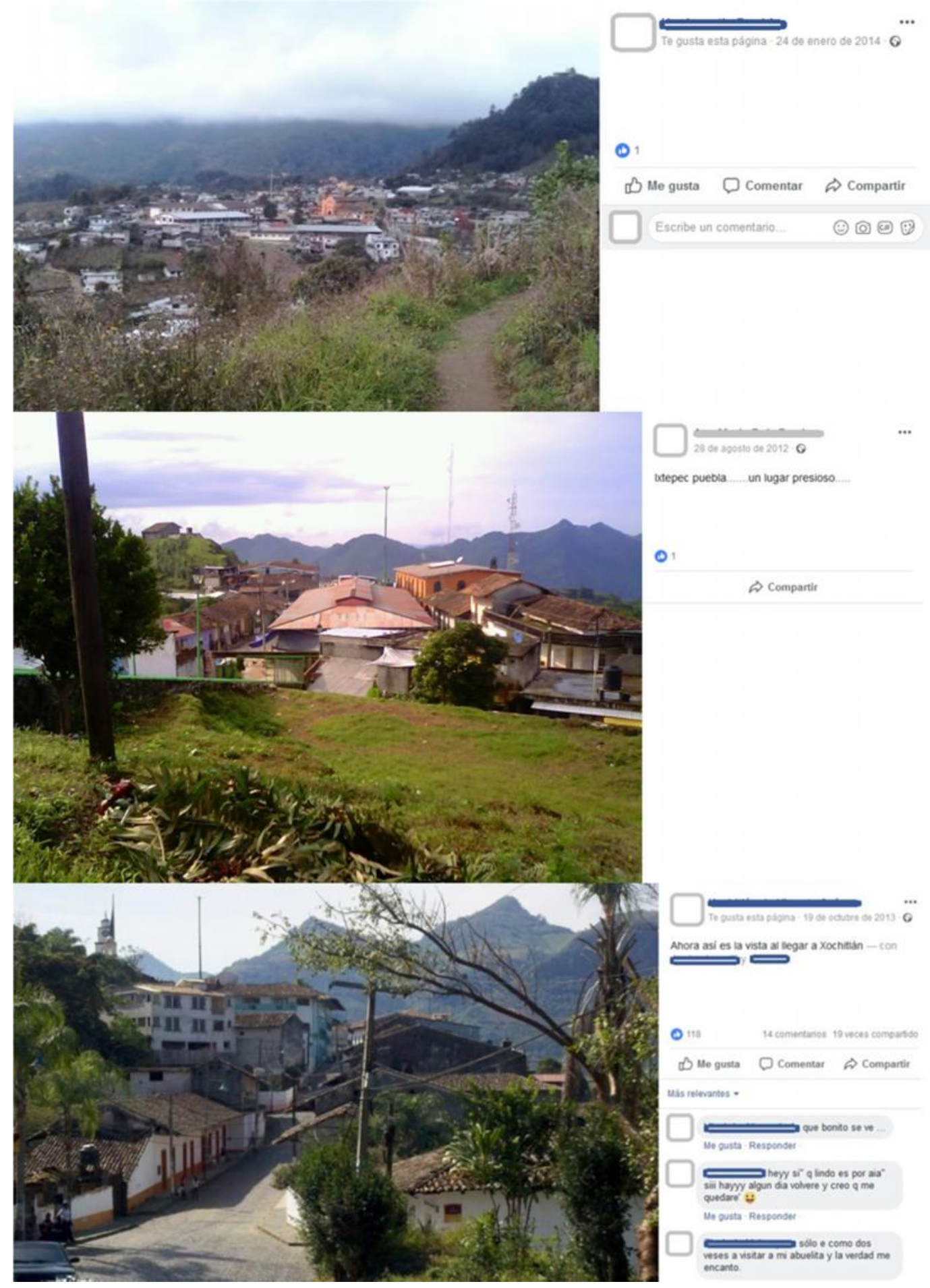

Fuente: Capturas de imagen propias a partir de perfiles de redes sociales. 
Imagen 4. Paisajes de la Sierra.
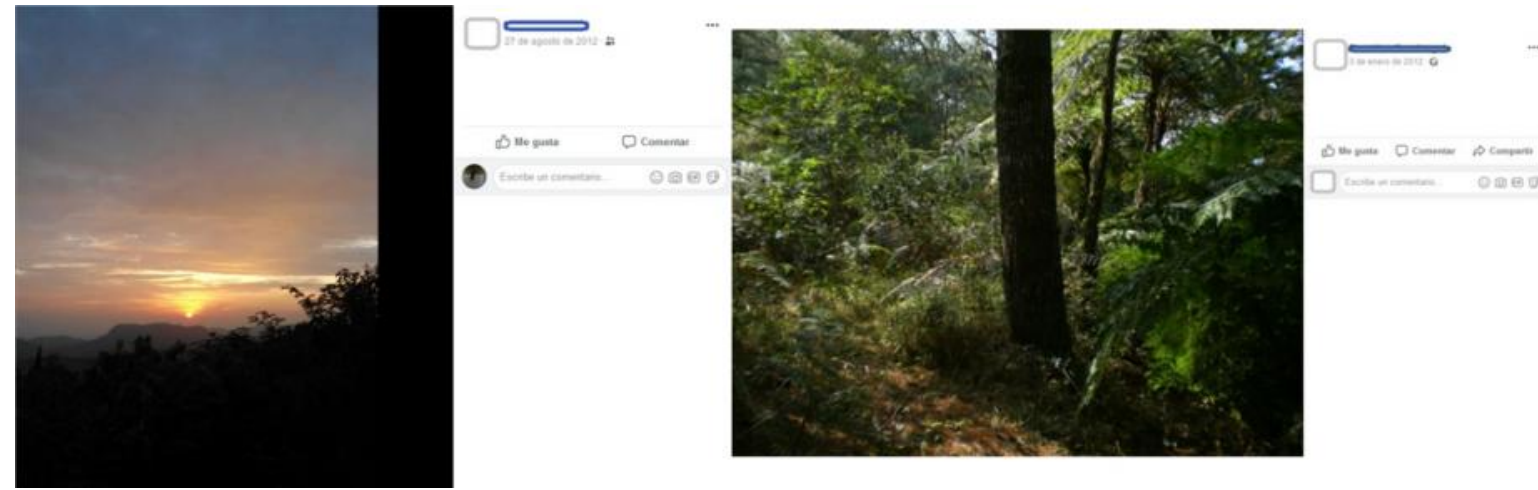

Fuente: Capturas de imagen propia a partir de los perfiles de redes sociales.

\section{Familia y comunicación}

Hay toda una serie de fotografías de reuniones y fiestas familiares que denotan su importancia entre los jóvenes. Generalmente esas fotografías se acompañan de comentarios y saludos realizados por otros parientes que han migrado a las ciudades, con lo que intentan trasladar la presencia de los ausentes. Al mismo tiempo, aquellas personas que están fuera del poblado también realizan publicaciones (fotografías o texto) en donde se indican sus lugares de residencia en ciudades de México y Estados Unidos, sentados en restaurantes, posando en parques o centros comerciales, realizando sus actividades laborales, etcétera. Relacionado a este tipo de contenidos destaca que en el ámbito familiar son las y los jóvenes quienes realizan la intermediación entre familiares para mantener comunicación, aunque sólo la que se realiza por redes sociales porque las llamadas telefónicas siguen predominando, más aún cuando ha incrementado la telefonía móvil.

\section{Jóvenes y tecnologías digitales}

En relación a la incorporación de las tecnologías digitales en los diferentes ámbitos de las vidas de los jóvenes en los poblados indígenas, ésta comienza con su asociación hacia lo educativo pues es en las escuelas donde les dejan tareas "a computadora" (escritos en procesador de textos e impresos) o que "investiguen en internet", hay cursos de informática en los programas educativos y, en algunos casos y poco a poco, equipos de cómputo para los cursos y para la realización de los trabajos escolares. Mucha de la demanda propiciada por las escuelas la van cubriendo los cibercafés, incluso dentro del gasto familiar se llega a contemplar lo relacionado a los "cibers". Por su parte, las y los jóvenes acuden a estos sitios de acceso a internet no sólo para realizar tareas escolares, sino que se conectan a internet para acceder a las redes sociales, principalmente Facebook desde donde se comunican con sus contactos o ver lo que han "publicado", escribir un comentario, y también 
para leer noticias locales, regionales, de entretenimiento. También, se conectan para publicar o subir alguna foto o video, para escribir un texto, esto es, generan contenido con el cual dinamizan la red social y, también, dinamizan sus ideas en torno a diferentes temas como los que se he presentado en el apartado anterior.

La apropiación de las tecnologías de información debe considerarse a partir de las condiciones materiales que posibilitan la conexión a, principalmente, internet. Junto a ello también influyen las personas mediadoras que según sus interrelaciones con los contextos locales pueden incentivar o detener la exploración de los servicios vinculados a internet y las tecnologías digitales. Pero, también hay otras influencias mássutiles, como lo son los medios de comunicación, por ejemplo, la televisión y la radio comercial al emitir constantes representaciones de lo juvenil vinculado a lo tecnológico y lo digital (URTEAGA; GARCÍA, 2015; WINOCUR, 2006). Lo cierto es que la materialidad de lo digital puede observarse en los poblados indígenas, en muchas de las pláticas relacionadas al uso de las tecnologías que tuve con los jóvenes, éstos me fueron indicando sus dispositivos digitales, los cuales incluían memorias USB, cables, teléfonos de tipo Smartphone, tabletas y computadoras portátiles.

Los ejemplos de temas que abarcan las representaciones locales realizadas por parte de jóvenes indígenas son elementos que consideraron en su momento significativo para publicarlo y compartirlo, para buscar interacciones entre sus contactos, son parte de sus mundos sociales (MADER, 2007). En este sentido, ponen en el espacio público de las redes sociales ideas que corresponden a sus antecedentes sociales y culturales, como aquellas vinculadas al pasado, el territorio, las tradiciones, a la comunidad, por indicar algunos ejemplos, las reelaboran y contrastan con elementos locales y externos generando representaciones propias de su juventud y etnicidad. Además, se trata de contenido propio que, como ya se ha mencionado, es lo que sostiene a los servicios de redes sociales.

El uso de las redes sociales y en general de internet entre los jóvenes indígenas es cada vez más amplio y cotidiano. En los poblados donde realicé el trabajo de campo los jóvenes utilizaban las tecnologías digitales todos los días en diferentes momentos. Sin embargo, eso no implicaba que prefirieran ese mundo digitalizado ni que requirieran conectarse todo el tiempo, más bien, correspondía a las actividades y los momentos específicos de sus vidas o de las necesidades de algún miembro de sus familias. Pero, si bien la desconexión puede ser una elección, en estos poblados indígenas suele estar incidida por la carencia de infraestructura que posibilite la continuidad y calidad de servicios generales, entre ellos la conectividad. 


\section{Conclusiones}

A partir de este breve repaso por las condiciones de conectividad en los poblados indígenas, donde son las generaciones jóvenes quienes se conectan a internet y utilizan las redes sociales, puedo señalar que las interacciones y el contenido que circula por los servicios de las redes sociales se conforma como una extensión y continuidad de otros espacios sociales, específicamente de aquellos vinculados a la juventud, en los que están negociándose las posibilidades de ser joven, indígena, y parte de una comunidad.

Los jóvenes dinamizan sus muros de Facebook (aunque podría ser cualquier otro servicio de redes sociales) a partir de generar contenidos en los que cruzan múltiples elementos. A grandes rasgos, y a partir del seguimiento con algunos contactos, puedo distinguir dos influencias, por un lado, los elementos localesdesde los cuales se realiza toda una serie de representaciones de las festividades, de las costumbres, de los territorios que habitan, de los lugares donde suelen reunirse y de las actividades que realizan (RAMOS, 2015b). Por otra parte, están los intereses que les van surgiendo por las relaciones que van estableciendo con otras personas o de los nuevos lugares de residencia, así, van ampliando sus gustos, perspectivas, y más importante, sus miradas hacia sus lugares de origen desde las cuales se pone en juego el arraigo y la identidad (RAMOS, 2015b).

La flexibilidad de los contenidos digitales que van produciendo sugiere que a pesar de que las personas jóvenes se van integrando en dinámicas distantes a sus pueblos, continúan circulando hacia y entre sus referentes locales primarios, aunque ya no sólo éstos. Estas interacciones e intercambios actuales inciden en el carácter dinámico de lo indígena, en donde lo digital puede considerarse como un aspecto inherente en las reelaboraciones étnicas en los tiempos actuales (PEREIRA, 2012).

Considero que la articulación que los jóvenes realizan entre bits junto con prácticas y relaciones sociales forma parte de una lógica de entender el mundo y de sus interacciones. En este sentido, mientras algunas personas, en su mayoría adultas, se tienen que ir adaptando y apropiando de las tecnologías que van surgiendo y las van asociando a sus actividades, hay otras personas, en específico jóvenes, que ya crecieron en un entorno donde se encuentran múltiples tecnologías en distintos ámbitos de la vida, por ejemplo, un telecentro público, la telefonía móvil, clases de informática en las escuelas, dispositivos más accesiblesen términos económicos, por señalar algunos ejemplos del entorno digital. Es decir, un entorno que se ha conformado socio-históricamente y que actualmente facilita la conjugación de lo digital con las y los jóvenes. Por ello, es parte de los referentes sociales y culturales locales. 
Finalmente, a partir de este trabajo surgieron algunas inquietudes para continuar explorando, una de ellas tiene relación con las dinámicas actuales que propician los dispositivos tipo Smartphone y la conectividad inalámbrica porque en el momento del trabajo de campo comenzaba a incrementar su adquisición y uso en los poblados. Un asunto similar se vincula con los usos de otros servicios de redes sociales, pues si bien Facebook continúa teniendo predominio, no era el único servicio que utilizaban los jóvenes indígenas, mucho menos aquellos que migran y tienen mayor conectividad en las ciudades. En general, están abiertas las vías a continuar explorando las definiciones y características de las juventudes indígenas a partir del agregado digital.

\section{Referencias}

ACOSTA NATES, P. A. Tecnologías de la información y la comunicación en poblaciones indígenas. Manizales: Universidad de Caldas, 2017.

BENNET, A.; ROBARDS, B. (Eds.). Mediated Youth Cultures. The internet, belonging and new cultural configurations. New York: Palgrave Macmillan, 2014.

BENNETT, S.; MATON, K.; KERVIN, L. The 'Digital Natives' Debate: A Critical Review of the Evidence. British J ournal of Educational Technology, v. 39, n. 5, 775-786, 2008.

BOYD, d. It's Complicated. The social lives of networked teens. New Haven: Yale University Press, 2014.

BUDKA, P. Indigenous media technology production in Northern Ontario, Canada. In: ERTLER, K. D.; LUTZ, H. (Eds.). Canada in Grainau / Le Canada à Grainau: A multidisciplinary survey of Canadian Studies after 30 years. Frankfurt: Peter Lang, 2009. p.63-74.

BURRELL, J. User Agency in the Middle Range: Rumors and the Reinvention of the Internet in Accra, Ghana. Science, Techonology \& Human Values, v. 36, n. 2, 139-159, 2011.

BURRELL, J. (2009). The Field Site as a Network: A Strategy for Locating Ethnographic Research. Field Methods, v. 21, n. 2, 181-199, 2009.

CLIFFORD, J. Varieties of Indigenous Experience: Diasporas, Homelands, Sovereignties. In: M. de la Cadena; O. Starn (Eds.). Indigenous Experience Today. Londres: Berg, 2007. p. 197-223. 
CORTÉS RIVERA, D.; HERNÁNDEZ, D.J uventud indígena en México. Una reflexiónepistemológica desde la sociología de las ausencias. Argumentos, n. 18, 149-176, 2016.

DOMÍ NGUEZ FIGAREDO, D. Escenarios híbridos, narrativas transmedia, etnografía expandida. Revista de Antropología Social, v. 21,197-215, 2012.

DUTTON, W. Society on the Line: information politics in the digital age. New York: Oxford University Press, 1999.

DYSON, L. E.; HENDRIKS, M.;GRANT S. Information technology and indigenous people. London: Idea, 2007.

FEENBERG, A.; FRIESEN, N. (ed.). (Re)Inventing the Internet. Critical Case Studies. Rotterdam: Sense Publishers, 2012.

FEIXA, C. De jóvenes, bandas y tribus. Antropología de la juventud. Barcelona: Arial, 1998.

GINSBURG, F. (2008). Rethinking the Digital Age. In:HESMONDHALGH,D.; TOYNBEE, J.(Eds.).The media and social theory. New York: Routledge, 2008. p. 127-144.

GI NSBURG, F.; ABU-LUGHOD, L.; LARKIN, B. I ntroduction. In: GINSBURG, F.; ABU-LUGHOD, L.; LARKIN, B. (Eds.). Media worlds: Anthropology on New Terrain. Los Angeles: University of California Press, 2002, p. 1-36.

HINE, C. Etnografía virtual. Barcelona: Editorial UOC, 2004.

KUMMELS, I. Transborder media spaces: Ayuujk videomaking between Mexico and the US.New York; Oxford: Bergham, 2017.

MADER, E. Encounters with Otavalo. Rituals identities, and the Internet. In:MURSIC, R.; REPIC, J. (Eds.). Places of Encounter: in memoriam Borut Brumen. Ljubljana: Zupanjceva Knjiznica, 2007. p. 221-239.

MEAD, M. Culture and Commitment: astudy of the generation gap. New York: Natural History Press, 1970.

MESEGUER GALVÁN, S. I maginarios de futuro de la juventud rural. Educación Superior Intercultural en la Sierra de Zongolica, Veracruz, México. 2012. 426 f. Tese (Doctorado en Antropología y Bienestar Social) - Departamento de Antropología Social, Universidad de Granada, 2012.

MILLER, D.; SLATER, D. The Internet: an ethnographic approach. Oxford: Berg, 2000. 
POSTILL, J. Localizing the Internet: an anthropological account. Oxford: Berghahn, 2011.

PRESKY, M. Digital natives, digital immigrants. On the Horizon, v. 9, n. 5, $1-6,2001$.

PEREIRA, E.S. Ciborgues indígen@s.br: a presença nativa no ciberespaço. São Paulo: Annablume, 2012.

PÉREZ RUÍZ, M.L. (Coord.). Jóvenes indígenas y globalización en América Latina. México: INAH, 2008.

PÉREZ RUÍZ, M.L. Retos para la investigación de los jóvenes indígenas. Alteridades, v. 21, n. 42, 65-75, 2011.

PÉREZ RUÍZ, M.L.;VALLADARES, L. (Coord.). Juventudes indígenas: de hip hop y protesta social en América Latina. México: INAH, 2014.

RAMOS MANCILLA, O. Dar vueltas al presente: juventud indígena y sus entramados en la era digital. In: VERDET, I.; ONGHENA, Y. (coord.). En tránsito: voces, acciones y (re)acciones. Barcelona: Monografías CIDOB, 2015a, p. 129-140.

RAMOS MANCILLA, O. Internet y pueblos indígenas de la Sierra Norte de Puebla, México. 2015b. 294 f. Tese (Doctorado en Antropología Social) Departamento de Antropología Social, Universidad de Barcelona, 2015b.

SLAVSKY, L. TIC's para jóvenes indígenas: una experiencia mapuche en la creación colectiva de contenidos culturales digitales. Quinto Sol, v. 11, 143-166, 2007.

SRINIVASAN, R. Indigenous, ethnic and cultural articulation of new media. International Journal of Cultural Studies, v. 9, n. 4, 497-518,2006.

SRIVINASAN, R. Whose global village? Rethinking how technologies shapes our world. New York: NYU Press, 2017.

URTEAGA CASTRO POZO, M. La construcción juvenil de la realidad: jóvenes mexicanos contemporáneos. México: Juan Pablos-UAM-I, 2011.

URTEAGA CASTRO POZO, M.; GARCÍ A ÁLVAREZ, L.F. Juventudes étnicas contemporáneas en Latinoamérica. Cuicuilco, v. 22, n. 62, 9-35, 2015.

VAN DEURSEN, A.;VAN DIJK, J. I nternet skills and the digital divide. New Media \& Society, v. 13, n. 6, 893-911, 2010. 
WINOCUR, R. Internet en la vida cotidiana de los jóvenes. Revista Mexicana de Sociología, v. 68, n. 3, 551-580, 2006.

ZAPATA CARDONA, C. A.; HOYOS AGUDELO, M. ¿Existeunacondicióndejuventudindígena? Nómadas, n. 23, 28-37,2005. 